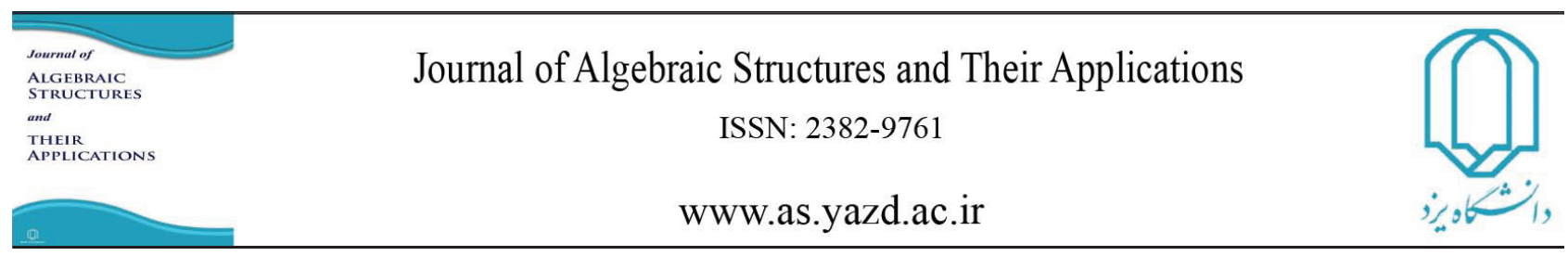

Algebraic Structures and Their Applications Vol. 5 No. 2 ( 2018 ) pp 23-34.

\title{
SPECTRA OF SOME NEW EXTENDED CORONA
}

\author{
MALIHEH TAJARROD AND TAHEREH SISTANI*
}

Communicated by A.R. Ashrafi

\begin{abstract}
For two graphs $\mathrm{G}$ and $\mathrm{H}$ with $n$ and $m$ vertices, the corona $\mathrm{G} \circ \mathrm{H}$ of $\mathrm{G}$ and $\mathrm{H}$ is the graph obtained by taking one copy of $\mathrm{G}$ and $n$ copies of $\mathrm{H}$ and then joining the $i^{t h}$ vertex of $\mathrm{G}$ to every vertex in the $i^{t h}$ copy of $\mathrm{H}$. The neighborhood corona $\mathrm{G} \star \mathrm{H}$ of $\mathrm{G}$ and $\mathrm{H}$ is the graph obtained by taking one copy of $\mathrm{G}$ and $n$ copies of $\mathrm{H}$ and joining every neighbor of the $i^{\text {th }}$ vertex of $\mathrm{G}$ to every vertex in the $i^{t h}$ copy of $\mathrm{H}$. In this paper, we define four new extensions of corona and neighborhood corona of two graphs $\mathrm{G}$ and $\mathrm{H}$; named the identity-extended corona, identity-extended neighborhood corona, neighborhood extended corona and neighborhood extended neighborhood corona and then determine the spectrum of their adjacency matrix, where $\mathrm{H}$ is a regular graph. As an application, we exhibit infinite families of integral graphs.
\end{abstract}

\section{INTRODUCTION}

All graphs considered in this paper are undirected and simple. Let G be a graph with vertex set $\mathrm{V}(\mathrm{G})=\left\{v_{1}, \ldots, v_{n}\right\}$ and edge set $\mathrm{E}(\mathrm{G})$. Let $\mathrm{M}=\mathrm{M}(\mathrm{G})$ be a $n \times n$ matrix associated to $\mathrm{G}$, DOI:http://dx.doi.org/10.29252/asta.5.2.23

MSC(2010): Primary: 05C50; Secondary: 05C76

Keywords: spectrum, corona, neighborhood corona, integral graphs.

Received: 23 August 2018, Accepted: 07 November 2018

$*$ Corresponding author

(C) 2018 Yazd University. 
the set of all the eigenvalues of $\mathrm{M}$ is called the M-spectrum of graph $\mathrm{G}$ which are the roots of the characteristic polynomial of $M$. In this paper, the characteristic polynomial of $M$ is denoted by $\varphi(\mathrm{M} ; x)=\operatorname{det}\left(x \mathrm{I}_{n}-\mathrm{M}\right)$, where $\mathrm{I}_{n}$ is the identity matrix of size $n$. The adjacency matrix of $\mathrm{G}$, denoted by $\mathrm{A}(\mathrm{G})=\left(a_{i j}\right)_{n \times n}$, is a $n \times n$ symmetric matrix in which $a_{i j}=1$ if $v_{i}$ is adjacent to $v_{j}$ and 0 otherwise. The graph $\mathrm{G}$ is integral if A-spectrum of $\mathrm{G}$ consists of only integers. $\mathrm{K}_{n}, \mathrm{~K}_{n, n}$ and $\mathrm{C}_{n}$ (just for $n=3,4,6$ ) are examples of integral graphs; see [3] for more details. Let $d_{i}=d_{\mathrm{G}}\left(v_{i}\right)$ be the degree of vertex $v_{i}$ in $\mathrm{G}$ and $\mathrm{D}(\mathrm{G})=\left(d_{i j}\right)_{n \times n}$ be the diagonal matrix such that $d_{i i}=d_{i}$ for each $1 \leq i \leq n$. The Laplacian matrix and the signless Laplacian matrix of $\mathrm{G}$ are defined as $\mathrm{L}(\mathrm{G})=\mathrm{D}(\mathrm{G})-\mathrm{A}(\mathrm{G})$ and $\mathrm{Q}(\mathrm{G})=\mathrm{D}(\mathrm{G})+\mathrm{A}(\mathrm{G})$, respectively.

So far, the various spectra of many graph operations such as the disjoint union, Cartesian product, Kronecker product, hierarchical product and subdivision-vertex and subdivision-edge

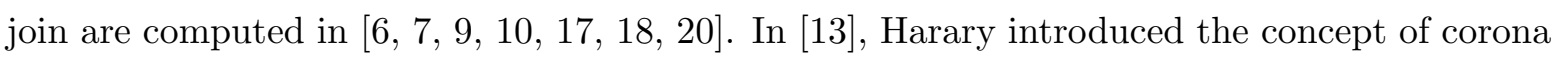
of a graph. Followed by, the A-spectrum, L-spectrum and Q-spectrum of the corona, edge corona, neighborhood corona, subdivision-vertex and subdivision-edge corona and subdivisionvertex and subdivision-edge neighborhood corona of two graphs $\mathrm{G}$ and $\mathrm{H}$ were computed in [4, 8, 12, 15, 19, 21, 222, 24]. Barik and Sahoo [5] have defined some more variations of double corona graphs and described their Laplacian spectrum. Recently, Adiga et al. [1, 2] have introduced some new extension of corona, neighborhood corona and edge corona of two graphs and computed their A-spectrum, L-spectrum and Q-spectrum. In [2:3], an extended corona product for weighted graphs is defined and then applying this generalized corona product and the reinforcement mechanism of edge weight in realistic networks, the authors introduced a simple generative model for heterogeneous weighted networks, which leads to rich topological and weighted properties.

In this paper, motivated by [T], we define four new extended corona of two graphs $G$ and $\mathrm{H}$ and determine their $\mathrm{A}$-spectrum where $\mathrm{H}$ is a regular graph. As an application, we exhibit infinite families of integral graphs.

\section{Preliminaries}

In this section, we need to state some results which will be used frequently later. Let $\mathrm{G}$ and $\mathrm{H}$ be two graphs on $n$ and $m$ vertices such that $\mathrm{V}(\mathrm{G})=\left\{v_{1}, v_{2}, \ldots, v_{n}\right\}$ and $\mathrm{V}(\mathrm{H})=$ $\left\{u_{1}, u_{2}, \ldots, u_{m}\right\}$. The corona $\mathrm{G} \circ \mathrm{H}$ of $\mathrm{G}$ and $\mathrm{H}$ is the graph obtained by taking one copy of $\mathrm{G}$ and $n$ copies of $\mathrm{H}$ and if $\mathrm{V}(\mathrm{G} \circ \mathrm{H})=\left\{v_{1_{1}}, \ldots, v_{1_{m}}, v_{2_{1}}, \ldots, v_{2_{m}}, \ldots, v_{n_{1}}, \ldots, v_{n_{m}}, v_{1}, \ldots, v_{n}\right\}$, then for each $i \in\{1,2, \ldots, n\}, v_{i}$ is adjacent to $v_{i_{k}}$ for each $k \in\{1,2, \ldots, m\}$ where $v_{i_{k}}$ is considered the same as $u_{k}$ in the $i^{\text {th }}$ copy of $\mathrm{H}$. The neighborhood corona $\mathrm{G} \star \mathrm{H}$ of two graphs $\mathrm{G}$ and $\mathrm{H}$ is the graph obtained by taking one copy of $\mathrm{G}$ and $n$ copies of $\mathrm{H}$ and if $\mathrm{V}(\mathrm{G} \star \mathrm{H})=\left\{v_{1_{1}}, \ldots, v_{1_{m}}, v_{2_{1}}, \ldots, v_{2_{m}}, \ldots, v_{n_{1}}, \ldots, v_{n_{m}}, v_{1}, \ldots, v_{n}\right\}$, then for each $i \in\{1,2, \ldots, n\}$, 
every neighbor of $v_{i}$ is adjacent to $v_{i_{k}}$ for each $k \in\{1,2, \ldots, m\}$, again $v_{i_{k}}$ is considered the same as $u_{k}$ in the $i^{t h}$ copy of $\mathrm{H}$. Let $\sigma$ be a permutation of $n$ items $\{1,2, \ldots, n\}$ which is written in the one-line notation as $\sigma(1), \sigma(2), \ldots, \sigma(n)$, that is, an ordered arrangement of $\{1,2, \ldots, n\}$. If for each $i \in\{1,2, \ldots, n\}, \sigma(i)=i$, we call $\sigma$ the identity permutation. Now, motivated by [I] and the concept of permutation, we define the identity-extended corona and identity-extended neighborhood corona as follows:

Definition 2.1. The identity-extended corona $I_{e x}(\mathrm{G} \circ \mathrm{H})$ of two graphs $\mathrm{G}$ and $\mathrm{H}$ is the graph obtained by taking the corona $\mathrm{G} \circ \mathrm{H}$ and joining the vertex $v_{i_{k}}$ of $i^{t h}$ copy of $\mathrm{H}$ to the vertex

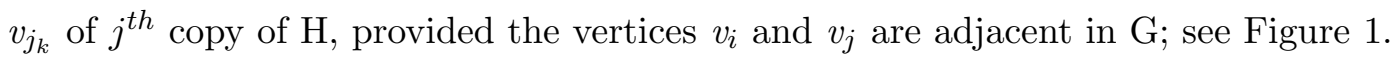

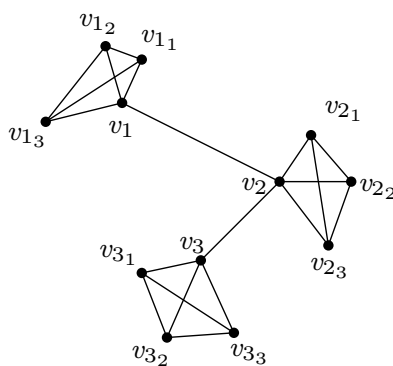

$\mathrm{P}_{3} \circ \mathrm{C}_{3}$

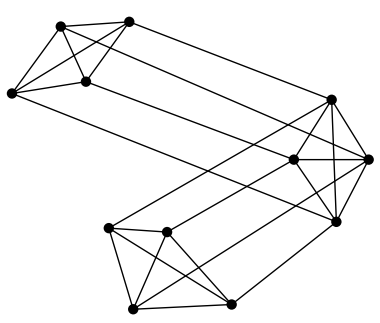

$I_{e x}\left(\mathrm{P}_{3} \circ \mathrm{C}_{3}\right)$

FiguRE 1. the corona and the identity-extended corona of two graphs $\mathrm{P}_{3}$ and $\mathrm{C}_{3}$

Definition 2.2. The identity-extended neighborhood corona $I_{e x}(\mathrm{G} \star \mathrm{H})$ of two graphs $\mathrm{G}$ and $\mathrm{H}$ is the graph obtained by taking the neighborhood corona $\mathrm{G} \star \mathrm{H}$ and joining the vertex $v_{i_{k}}$ of $i^{\text {th }}$ copy of $\mathrm{H}$ to the vertex $v_{j_{k}}$ of $j^{t h}$ copy of $\mathrm{H}$, provided the vertices $v_{i}$ and $v_{j}$ are adjacent in G; see Figure [2].

The following definitions are two other extensions of corona and neighborhood corona of graphs.

Definition 2.3. The neighborhood extended corona $N_{e x}(\mathrm{G} \circ \mathrm{H})$ of two graphs $\mathrm{G}$ and $\mathrm{H}$ is the graph obtained by taking the corona $\mathrm{G} \circ \mathrm{H}$ and joining the vertex $v_{i_{k}}$ of $i^{t h}$ copy of $\mathrm{H}$ to the vertices $v_{j_{l}}$ of $j^{\text {th }}$ copy of $\mathrm{H}$, provided the vertices $v_{i}$ and $v_{j}$ are adjacent in $\mathrm{G}$ and $u_{k}$ and $u_{l}$ are adjacent in $\mathrm{H}$; see Figure $\mathbf{3}$.

Definition 2.4. The neighborhood extended neighborhood corona $N_{e x}(\mathrm{G} \star \mathrm{H})$ of two graphs $\mathrm{G}$ and $\mathrm{H}$ is the graph obtained by taking the neighborhood corona $\mathrm{G} \star \mathrm{H}$ and joining the vertex $v_{i_{k}}$ of $i^{t h}$ copy of $\mathrm{H}$ to the vertices $v_{j_{l}}$ of $j^{\text {th }}$ copy of $\mathrm{H}$, provided the vertices $v_{i}$ and $v_{j}$ are adjacent in $\mathrm{G}$ and $u_{k}$ and $u_{l}$ are adjacent in $\mathrm{H}$; see Figure 3 . 


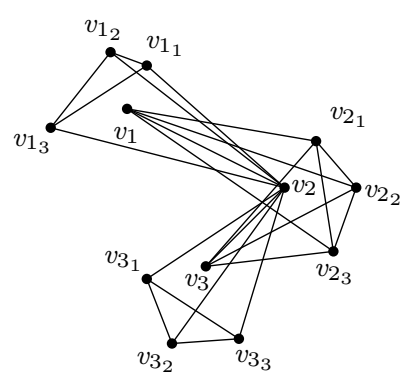

$$
\mathrm{P}_{3} \star \mathrm{C}_{3}
$$

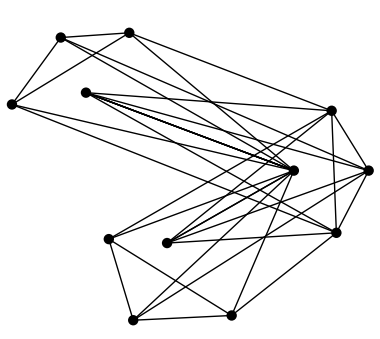

$I_{e x}\left(\mathrm{P}_{3} \star \mathrm{C}_{3}\right)$

FIGURE 2. the neighborhood corona and the identity-extended neighborhood corona of two graphs $\mathrm{P}_{3}$ and $\mathrm{C}_{3}$

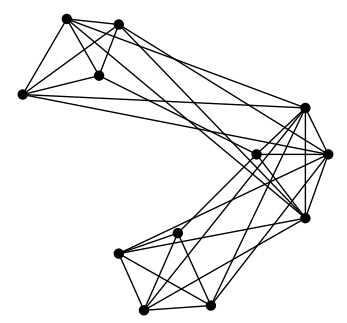

$N_{e x}\left(\mathrm{P}_{3} \circ \mathrm{C}_{3}\right)$

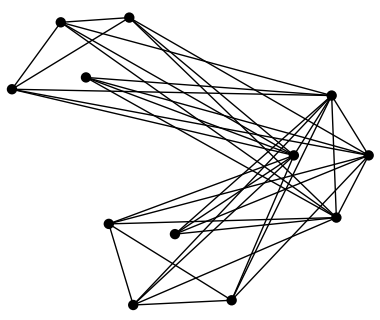

$N_{e x}\left(\mathrm{P}_{3} \star \mathrm{C}_{3}\right)$

Figure 3. Two neighborhood extended corona of two graphs $\mathrm{P}_{3}$ and $\mathrm{C}_{3}$

In this paper, suppose that $\mathrm{J}=\left[e_{i j}\right]_{s \times t}$ denotes the $s \times t$ matrix with all entries equal to one and $\mathrm{J}_{s \times t}^{\prime}$ obtained by replacing every entry of $\mathrm{J}_{s \times t}$ by 0 except $e_{11}$. The Kronecker product $\mathrm{A} \otimes \mathrm{B}$ of two matrices $\mathrm{A}=\left(a_{i j}\right)_{n \times m}$ and $\mathrm{B}=\left(b_{i j}\right)_{p \times q}$ is the $n p \times m q$ matrix obtained from $\mathrm{A}$ by replacing each entry $a_{i j}$ by $a_{i j} \mathrm{~B}$, see [14]. For matrices A, B, C and D, $(\mathrm{A} \otimes \mathrm{B})(\mathrm{C} \otimes \mathrm{D})=$ $\mathrm{AC} \otimes \mathrm{BD}$ whenever the products $\mathrm{AC}$ and $\mathrm{BD}$ exist.

The M-coronal $\Gamma_{\mathrm{M}}(x)$ of an $n \times n$ matrix $\mathrm{M}$ is defined to be the sum of the entries of the matrix $\left(x \mathrm{I}_{n}-\mathrm{M}\right)^{-1}$, that is

$$
\Gamma_{\mathrm{M}}(x)=\mathbf{1}_{n}^{T}\left(x \mathrm{I}_{n}-\mathrm{M}\right)^{-1} \mathbf{1}_{n},
$$

where $\mathbf{1}_{n}$ denotes the column vector of dimension $n$ with all the components equal to one; see $[22]$.

Proposition $2.5([8])$. Let $\mathrm{M}$ be an $n \times n$ matrix with each row sum equal to constant $c$, then

$$
\Gamma_{\mathrm{M}}(x)=\frac{n}{x-c} .
$$


Proposition $2.6([20])$. Let A be an $n \times n$ real matrix, then

$$
\operatorname{det}\left(x \mathrm{I}_{n}-\mathrm{A}-\alpha \mathrm{J}_{n \times n}\right)=\left(1-\alpha \Gamma_{\mathrm{A}}(x)\right) \cdot \operatorname{det}\left(x \mathrm{I}_{n}-\mathrm{A}\right) .
$$

Proposition 2.7 ([25]). Let $\mathrm{M}_{1}, \mathrm{M}_{2}, \mathrm{M}_{3}$ and $\mathrm{M}_{4}$ be respectively $p \times p, p \times q, q \times p$ and $q \times q$ matrices with $\mathrm{M}_{1}$ and $\mathrm{M}_{4}$ invertible. Then

$$
\begin{aligned}
\operatorname{det}\left(\left[\begin{array}{ll}
\mathrm{M}_{1} & \mathrm{M}_{2} \\
\mathrm{M}_{3} & \mathrm{M}_{4}
\end{array}\right]\right) & =\operatorname{det}\left(\mathrm{M}_{4}\right) \cdot \operatorname{det}\left(\mathrm{M}_{1}-\mathrm{M}_{2} \mathrm{M}_{4}^{-1} \mathrm{M}_{3}\right) \\
& =\operatorname{det}\left(\mathrm{M}_{1}\right) \cdot \operatorname{det}\left(\mathrm{M}_{4}-\mathrm{M}_{3} \mathrm{M}_{1}^{-1} \mathrm{M}_{2}\right)
\end{aligned}
$$

where $\mathrm{M}_{1}-\mathrm{M}_{2} \mathrm{M}_{4}^{-1} \mathrm{M}_{3}$ and $\mathrm{M}_{4}-\mathrm{M}_{3} \mathrm{M}_{1}^{-1} \mathrm{M}_{2}$ are called the Schur complements of $\mathrm{M}_{4}$ and $\mathrm{M}_{1}$, respectively.

In this paper, we apply the Laplace's expansion of a determinants by complementry minors several times; For more details, one can see page 36 of []].

\section{SPeCtra of THE IDENTITY-EXTENDED CORONA AND IDENTITY-EXTENDED}

\section{NEIGHBORHOOD CORONA}

In this section, $\mathrm{G}$ is an arbitrary graph with $n$ vertices and $\mathrm{H}$ is a $r$-regular graph with $m$ vertices. To write the adjacency matrix of the identity-extended corona and identity-extended neighborhood corona, we maintain the arrangement of vertices in $\mathrm{V}(\mathrm{G} \circ \mathrm{H})$. First, we present the spectra of $I_{e x}(\mathrm{G} \circ \mathrm{H})$.

Theorem 3.1. The adjacency spectrum of $I_{e x}(\mathrm{G} \circ \mathrm{H})$ is:

i) $\lambda_{i}(\mathrm{H})+\lambda_{j}(\mathrm{G})$ for $i=2, \ldots, m$ and $j=1, \ldots, n$;

ii) $\left(2 \lambda_{j}(\mathrm{G})+r \pm \sqrt{r^{2}+4 m}\right) / 2$ for $j=1, \ldots, n$.

Proof. The adjacency matrix of $I_{e x}(\mathrm{G} \circ \mathrm{H})$ can be expressed in the following form:

$$
\mathrm{A}\left(I_{e x}(\mathrm{G} \circ \mathrm{H})\right)=\left[\begin{array}{cc}
\mathrm{I}_{n} \otimes \mathrm{A}(\mathrm{H})+\mathrm{A}(\mathrm{G}) \otimes \mathrm{I}_{m} & \mathrm{I}_{n} \otimes \mathrm{J}_{m \times 1} \\
\mathrm{I}_{n} \otimes \mathrm{J}_{1 \times m} & \mathrm{~A}(\mathrm{G})
\end{array}\right]
$$

$\mathrm{A}(\mathrm{H})$ is a real Hermitian matrix, so $\mathrm{A}(\mathrm{H})$ is orthogonally diagonalizable and Since $\mathrm{H}$ is $r$ regular, $\mathrm{A}(\mathrm{H})=\mathrm{PD}(\mathrm{H}) \mathrm{P}^{\mathrm{T}}$ where $\mathrm{D}(\mathrm{H})=\operatorname{diag}\left(r, \lambda_{2}(\mathrm{H}), \ldots, \lambda_{m}(\mathrm{H})\right)$ and $\mathrm{P}$ is a square matrix 
of order $m$ such that its first column vector is $\frac{1}{\sqrt{m}}(1,1, \ldots, 1)$ and $\mathrm{P}^{\mathrm{T}} \mathrm{P}=\mathrm{I}_{\mathrm{m}}$. Therefore,

$$
\begin{aligned}
\mathrm{A}\left(I_{e x}(\mathrm{G} \circ \mathrm{H})\right) & =\left[\begin{array}{cc}
\mathrm{I}_{n} \otimes \mathrm{PD}(\mathrm{H}) \mathrm{P}^{\mathrm{T}}+\mathrm{A}(\mathrm{G}) \otimes \mathrm{I}_{m} & \mathrm{I}_{n} \otimes \mathrm{J}_{m \times 1} \\
\mathrm{I}_{n} \otimes \mathrm{J}_{1 \times m} & \mathrm{~A}(\mathrm{G})
\end{array}\right] \\
& =\left[\begin{array}{cc}
\mathrm{I}_{n} \otimes \mathrm{P} & 0 \\
0 & \mathrm{I}_{n}
\end{array}\right]\left[\begin{array}{cc}
\mathrm{I}_{n} \otimes \mathrm{D}(\mathrm{H})+\mathrm{A}(\mathrm{G}) \otimes \mathrm{I}_{m} & \mathrm{I}_{n} \otimes \mathrm{P}^{\mathrm{T}} \mathrm{J}_{m \times 1} \\
\mathrm{I}_{n} \otimes \mathrm{J}_{1 \times m} \mathrm{P} & \mathrm{A}(\mathrm{G})
\end{array}\right]\left[\begin{array}{cc}
\mathrm{I}_{n} \otimes \mathrm{P}^{\mathrm{T}} & 0 \\
0 & \mathrm{I}_{n}
\end{array}\right] \\
& =\left[\begin{array}{cc}
\mathrm{I}_{n} \otimes \mathrm{P} & 0 \\
0 & \mathrm{I}_{n}
\end{array}\right]\left[\begin{array}{cc}
\mathrm{I}_{n} \otimes \mathrm{D}(\mathrm{H})+\mathrm{A}(\mathrm{G}) \otimes \mathrm{I}_{m} & \mathrm{I}_{n} \otimes \sqrt{m} \mathrm{~J}_{m \times 1}^{\prime} \\
\mathrm{I}_{n} \otimes \sqrt{m} \mathrm{~J}^{\prime} & \mathrm{A}(\mathrm{G})
\end{array}\right]\left[\begin{array}{cc}
\mathrm{I}_{n} \otimes \mathrm{P}^{\mathrm{T}} & 0 \\
0 & \mathrm{I}_{n}
\end{array}\right] .
\end{aligned}
$$

So $\mathrm{A}\left(I_{e x}(\mathrm{G} \circ \mathrm{H})\right)$ is similar to

$$
\mathrm{B}_{0}=\left[\begin{array}{cc}
\mathrm{I}_{n} \otimes \mathrm{D}(\mathrm{H})+\mathrm{A}(\mathrm{G}) \otimes \mathrm{I}_{m} & \mathrm{I}_{n} \otimes \sqrt{m} \mathrm{~J}_{m \times 1}^{\prime} \\
\mathrm{I}_{n} \otimes \sqrt{m} \mathrm{~J}^{\prime}{ }_{1 \times m} & \mathrm{~A}(\mathrm{G})
\end{array}\right],
$$

and

$$
\begin{aligned}
\operatorname{det}\left(x \mathrm{I}-\mathrm{A}\left(\mathrm{I}_{e x}(\mathrm{G} \circ \mathrm{H})\right)\right) & =\operatorname{det}\left(x \mathrm{I}-\mathrm{B}_{0}\right) \\
& =\operatorname{det}\left[\begin{array}{cc}
\mathrm{I}_{n} \otimes\left(x \mathrm{I}_{m}-\mathrm{D}(\mathrm{H})\right)-\mathrm{A}(\mathrm{G}) \otimes \mathrm{I}_{m} & -\mathrm{I}_{n} \otimes \sqrt{m} \mathrm{~J}_{m \times 1}^{\prime} \\
-\mathrm{I}_{n} \otimes \sqrt{m} \mathrm{~J}^{\prime}{ }_{1 \times m} & x \mathrm{I}_{n}-\mathrm{A}(\mathrm{G})
\end{array}\right] .
\end{aligned}
$$

Using the Laplace's expansion of $\operatorname{det}\left(x \mathrm{I}-\mathrm{B}_{0}\right)$ by $(m i+2),(m i+3), \ldots,(m i+m)^{t h}$ columns for $i=0,1, \ldots, n-1$, we see that $\operatorname{det}\left(x \mathrm{I}-\mathrm{B}_{0}\right)=\mathrm{M} \cdot \mathrm{M}_{1}$ where $\mathrm{M}=\operatorname{det}\left(\mathrm{B}_{1}\right)$ and $\mathrm{B}_{1}=$ $\mathrm{I}_{n} \otimes \operatorname{diag}\left(x-\lambda_{2}(\mathrm{H}), \ldots, x-\lambda_{m}(\mathrm{H})\right)-\left(\mathrm{A}(\mathrm{G}) \otimes \mathrm{I}_{m-1}\right)$, and also

$$
\mathrm{M}_{1}=\operatorname{det}\left[\begin{array}{cc}
(x-r) \mathrm{I}_{n}-\mathrm{A}(\mathrm{G}) & -\sqrt{m} \mathrm{I}_{n} \\
-\sqrt{m} \mathrm{I}_{n} & x \mathrm{I}_{n}-\mathrm{A}(\mathrm{G})
\end{array}\right]
$$

is the complementary minor of M. Again expanding $\operatorname{det}\left(\mathrm{B}_{1}\right)$ by Laplace 's method in the similar way and continuing this method $(m-2)$ times, we have

$$
\begin{aligned}
\operatorname{det}\left(\mathrm{B}_{1}\right) & =\prod_{i=2}^{m} \operatorname{det}\left(\left(x-\lambda_{i}(\mathrm{H})\right) \mathrm{I}_{n}-\mathrm{A}(\mathrm{G})\right) \\
& =\prod_{i=2}^{m} \prod_{j=1}^{n}\left(x-\lambda_{i}(\mathrm{H})-\lambda_{j}(\mathrm{G})\right) \\
& =\prod_{\substack{2 \leqslant i \leqslant m \\
1 \leqslant j \leqslant n}}\left(x-\left(\lambda_{i}(\mathrm{H})+\lambda_{j}(\mathrm{G})\right)\right) .
\end{aligned}
$$

Again $A(G)$ is orthogonally diagonalizable, so we can easily see that

$$
\mathrm{M}_{1}=\operatorname{det}\left[\begin{array}{cc}
(x-r) \mathrm{I}_{n}-\mathrm{D}(\mathrm{G}) & -\sqrt{m} \mathrm{I}_{n} \\
-\sqrt{m} \mathrm{I}_{n} & x \mathrm{I}_{n}-\mathrm{D}(\mathrm{G})
\end{array}\right] .
$$


By Proposition [2.],

$$
\begin{aligned}
\mathrm{M}_{1} & =\operatorname{det}\left(x \mathrm{I}_{n}-\mathrm{D}(\mathrm{G})\right) \cdot \operatorname{det}\left((x-r) \mathrm{I}_{n}-\mathrm{D}(\mathrm{G})-m\left(x \mathrm{I}_{n}-\mathrm{D}(\mathrm{G})\right)^{-1}\right) \\
& =\operatorname{det}\left(x \mathrm{I}_{n}-\mathrm{D}(\mathrm{G})\right) \cdot \prod_{i=1}^{n}\left(x-r-\lambda_{i}(\mathrm{G})-\frac{m}{x-\lambda_{i}(\mathrm{G})}\right) \\
& =\prod_{i=1}^{n}\left(x-\lambda_{i}(\mathrm{G})\right) \cdot \prod_{i=1}^{n} \frac{x^{2}-\left(2 \lambda_{i}(\mathrm{G})+r\right) x+\lambda_{i}^{2}(\mathrm{G})+r \lambda_{i}(\mathrm{G})-m}{x-\lambda_{i}(\mathrm{G})} \\
& =\prod_{i=1}^{n}\left(x^{2}-\left(2 \lambda_{i}(\mathrm{G})+r\right) x+\left(\lambda_{i}^{2}(\mathrm{G})+r \lambda_{i}(\mathrm{G})-m\right)\right) .
\end{aligned}
$$

Finally,

$$
\begin{aligned}
\operatorname{det}\left(x \mathrm{I}-\mathrm{A}\left(I_{e x}(\mathrm{G} \circ \mathrm{H})\right)\right)= & \prod_{\substack{2 \leqslant i \leqslant m \\
1 \leqslant j \leqslant n}}\left(x-\left(\lambda_{i}(\mathrm{H})+\lambda_{j}(\mathrm{G})\right)\right) \\
& \cdot \prod_{i=1}^{n}\left(x^{2}-\left(2 \lambda_{i}(\mathrm{G})+r\right) x+\left(\lambda_{i}^{2}(\mathrm{G})+r \lambda_{i}(\mathrm{G})-m\right)\right) .
\end{aligned}
$$

Now, we present the spectra of the identity-extended neighborhood corona $I_{e x}(\mathrm{G} \star \mathrm{H})$ of two graphs $\mathrm{G}$ and $\mathrm{H}$, where $\mathrm{H}$ is regular.

Theorem 3.2. The adjacency spectrum of $I_{e x}(\mathrm{G} \star \mathrm{H})$ is as follows:

i) $\lambda_{i}(\mathrm{H})+\lambda_{j}(\mathrm{G})$ for $i=2, \ldots, m$ and $j=1, \ldots, n$;

ii) $\left(2 \lambda_{j}(\mathrm{G})+r \pm \sqrt{r^{2}+4 m \lambda_{j}^{2}(\mathrm{G})}\right) / 2$ for $j=1, \ldots, n$.

Proof. The adjacency matrix of $I_{e x}(\mathrm{G} \star \mathrm{H})$ can be written as:

$$
\mathrm{A}\left(I_{e x}(\mathrm{G} \star \mathrm{H})\right)=\left[\begin{array}{cc}
\mathrm{I}_{n} \otimes \mathrm{A}(\mathrm{H})+\mathrm{A}(\mathrm{G}) \otimes \mathrm{I}_{m} & \mathrm{~A}(\mathrm{G}) \otimes \mathrm{J}_{m \times 1} \\
\mathrm{~A}(\mathrm{G}) \otimes \mathrm{J}_{1 \times m} & \mathrm{~A}(\mathrm{G})
\end{array}\right] .
$$

Similar to the proof of Theorem $\left[\begin{array}{l}\mathrm{B} \\ \mathrm{D}\end{array}, \mathrm{A}\left(I_{e x}(\mathrm{G} \star \mathrm{H})\right)\right.$ is similar to

$$
\mathrm{B}_{0}=\left[\begin{array}{cc}
\mathrm{I}_{n} \otimes \mathrm{D}(\mathrm{H})+\mathrm{A}(\mathrm{G}) \otimes \mathrm{I}_{m} & \mathrm{~A}(\mathrm{G}) \otimes \sqrt{m} \mathrm{~J}_{m \times 1}^{\prime} \\
\mathrm{A}(\mathrm{G}) \otimes \sqrt{m} \mathrm{~J}^{\prime}{ }_{1 \times m} & \mathrm{~A}(\mathrm{G})
\end{array}\right],
$$

and

$$
\begin{aligned}
& \operatorname{det}\left(x \mathrm{I}-\mathrm{A}\left(I_{e x}(\mathrm{G} \star \mathrm{H})\right)\right)=\operatorname{det}\left(x \mathrm{I}-\mathrm{B}_{0}\right) \\
& =\operatorname{det}\left[\begin{array}{cc}
\mathrm{I}_{n} \otimes\left(x \mathrm{I}_{m}-\mathrm{D}(\mathrm{H})\right)-\mathrm{A}(\mathrm{G}) \otimes \mathrm{I}_{m} & -\mathrm{A}(\mathrm{G}) \otimes \sqrt{m} \mathrm{~J}_{m \times 1}^{\prime} \\
-\mathrm{A}(\mathrm{G}) \otimes \sqrt{m} \mathrm{~J}^{\prime}{ }_{1 \times m} & x \mathrm{I}_{n}-\mathrm{A}(\mathrm{G})
\end{array}\right] .
\end{aligned}
$$


Again similar to the proof of Theorem B.]; using the Laplace's expansion,

$$
\begin{aligned}
\operatorname{det}\left(x \mathrm{I}-\mathrm{A}\left(I_{e x}(\mathrm{G} \star \mathrm{H})\right)\right)= & \prod_{\substack{2 \leqslant i \leqslant m \\
1 \leqslant j \leqslant n}}\left(x-\left(\lambda_{i}(\mathrm{H})+\lambda_{j}(\mathrm{G})\right)\right) \\
& \cdot \operatorname{det}\left[\begin{array}{cc}
(x-r) \mathrm{I}_{n}-\mathrm{A}(\mathrm{G}) & -\sqrt{m} \mathrm{~A}(\mathrm{G}) \\
-\sqrt{m} \mathrm{~A}(\mathrm{G}) & x \mathrm{I}_{n}-\mathrm{A}(\mathrm{G})
\end{array}\right] .
\end{aligned}
$$

$A(G)$ is orthogonally diagonalizable, so we can easily see that

$$
\begin{aligned}
\operatorname{det}\left(x \mathrm{I}-\mathrm{A}\left(I_{e x}(\mathrm{G} \star \mathrm{H})\right)\right)= & \prod_{\substack{2 \leqslant i \leqslant m \\
1 \leqslant j \leqslant n}}\left(x-\left(\lambda_{i}(\mathrm{H})+\lambda_{j}(\mathrm{G})\right)\right) \\
& \cdot \operatorname{det}\left[\begin{array}{cc}
(x-r) \mathrm{I}_{n}-\mathrm{D}(\mathrm{G}) & -\sqrt{m} \mathrm{D}(\mathrm{G}) \\
-\sqrt{m} \mathrm{D}(\mathrm{G}) & x \mathrm{I}_{n}-\mathrm{D}(\mathrm{G})
\end{array}\right] \\
= & \prod_{\substack{2 \leqslant i \leqslant m \\
1 \leqslant j \leqslant n}}\left(x-\left(\lambda_{i}(\mathrm{H})+\lambda_{j}(\mathrm{G})\right)\right) \\
& \cdot \prod_{i=1}^{n}\left(x^{2}-\left(2 \lambda_{i}(\mathrm{G})+r\right) x+r \lambda_{i}(\mathrm{G})+(1-m) \lambda_{i}^{2}(\mathrm{G})\right) .
\end{aligned}
$$

\section{Spectra of the neighborhood EXtended CORONA AND Neighborhood EXtended}

\section{NEIGHBORHOOD CORONA}

In this section, we present the spectra of the neighborhood extended corona $N_{e x}(\mathrm{G} \circ \mathrm{H})$ and the neighborhood extended neighborhood corona $N_{e x}(\mathrm{G} \star \mathrm{H})$ of two graphs $\mathrm{G}$ and $\mathrm{H}$ where $\mathrm{G}$ is an arbitrary $n$-vertices and $\mathrm{H}$ is a $r$-regular $m$-vertices graph. To write the adjacency matrix of these two operations, we maintain the arrangement of vertices in $V(G \star H)$.

Theorem 4.1. The adjacency spectrum of $N_{e x}(\mathrm{G} \circ \mathrm{H})$ is:

i) $\lambda_{i}(\mathrm{H})\left(1+\lambda_{j}(\mathrm{G})\right)$ for $i=2, \ldots, m$ and $j=1, \ldots, n$;

ii) $\left((r+1) \lambda_{j}(\mathrm{G})+r \pm \sqrt{\left((r-1) \lambda_{j}(\mathrm{G})+r\right)^{2}+4 m}\right) / 2$ for $j=1, \ldots, n$.

Proof. The adjacency matrix of $N_{e x}(\mathrm{G} \circ \mathrm{H})$ can be expressed in the following form:

$$
\mathrm{A}\left(N_{e x}(\mathrm{G} \circ \mathrm{H})\right)=\left[\begin{array}{cc}
\left(\mathrm{I}_{n}+\mathrm{A}(\mathrm{G})\right) \otimes \mathrm{A}(\mathrm{H}) & \mathrm{I}_{n} \otimes \mathrm{J}_{m \times 1} \\
\mathrm{I}_{n} \otimes \mathrm{J}_{1 \times m} & \mathrm{~A}(\mathrm{G})
\end{array}\right] .
$$

$\mathrm{A}(\mathrm{H})$ is a real Hermitian matrix and $\mathrm{H}$ is $r$-regular, so we have $\mathrm{A}(\mathrm{H})=\mathrm{PD}(\mathrm{H}) \mathrm{P}^{\mathrm{T}}$ where $\mathrm{A}(\mathrm{H})=\operatorname{diag}\left(r, \lambda_{2}(\mathrm{H}), \ldots, \lambda_{m}(\mathrm{H})\right)$ and $\mathrm{P}$ is a square matrix of order $m$ such that its first 
column vector is $\frac{1}{\sqrt{m}}(1,1, \ldots, 1)$ and $\mathrm{P}^{\mathrm{T}} \mathrm{P}=\mathrm{I}_{\mathrm{m}}$. Therefore,

$$
\mathrm{A}\left(N_{e x}(\mathrm{G} \circ \mathrm{H})\right)=\left[\begin{array}{cc}
\mathrm{I}_{n} \otimes \mathrm{P} & 0 \\
0 & \mathrm{I}_{n}
\end{array}\right]\left[\begin{array}{cc}
\left(\mathrm{I}_{n}+\mathrm{A}(\mathrm{G})\right) \otimes \mathrm{D}(\mathrm{H}) & \mathrm{I}_{n} \otimes \sqrt{m} \mathrm{~J}_{m \times 1}^{\prime} \\
\mathrm{I}_{n} \otimes \sqrt{m} \mathrm{~J}^{\prime}{ }_{1 \times m} & \mathrm{~A}(\mathrm{G})
\end{array}\right]\left[\begin{array}{cc}
\mathrm{I}_{n} \otimes \mathrm{P}^{\mathrm{T}} & 0 \\
0 & \mathrm{I}_{n}
\end{array}\right]
$$

So $\mathrm{A}\left(N_{e x}(\mathrm{G} \circ \mathrm{H})\right)$ is similar to

$$
\mathrm{B}_{0}=\left[\begin{array}{cc}
\left(\mathrm{I}_{n}+\mathrm{A}(\mathrm{G})\right) \otimes \mathrm{D}(\mathrm{H}) & \mathrm{I}_{n} \otimes \sqrt{m} \mathrm{~J}_{m \times 1}^{\prime} \\
\mathrm{I}_{n} \otimes \sqrt{m} \mathrm{~J}^{\prime}{ }_{1 \times m} & \mathrm{~A}(\mathrm{G})
\end{array}\right]
$$

and

$$
\operatorname{det}\left(x \mathrm{I}-\mathrm{A}\left(N_{e x}(\mathrm{G} \circ \mathrm{H})\right)\right)=\operatorname{det}\left(x \mathrm{I}-\mathrm{B}_{0}\right) .
$$

Now, use the Laplace's expansion of $\operatorname{det}\left(x \mathrm{I}-\mathrm{B}_{0}\right)$ by $(m i+2),(m i+3), \ldots,(m i+m)^{t h}$ columns for $i=0,1, \ldots, n-1$. We have

$$
\operatorname{det}\left(x \mathrm{I}-\mathrm{B}_{0}\right)=\operatorname{det}\left(\mathrm{B}_{1}\right) \cdot \mathrm{M}_{1},
$$

where $\mathrm{B}_{1}=\mathrm{I}_{n} \otimes \operatorname{diag}\left(x-\lambda_{2}(\mathrm{H}), \ldots, x-\lambda_{m}(\mathrm{H})\right)-\left(\mathrm{A}(\mathrm{G}) \otimes \operatorname{diag}\left(\lambda_{2}(\mathrm{H}), \ldots, \lambda_{m}(\mathrm{H})\right)\right)$ and

$$
\mathrm{M}_{1}=\operatorname{det}\left[\begin{array}{cc}
(x-r) \mathrm{I}_{n}-r \mathrm{~A}(\mathrm{G}) & -\sqrt{m} \mathrm{I}_{n} \\
-\sqrt{m} \mathrm{I}_{n} & x \mathrm{I}_{n}-\mathrm{A}(\mathrm{G})
\end{array}\right]
$$

is the complementary minor of $\operatorname{det}\left(\mathrm{B}_{1}\right)$. Again expanding $\operatorname{det}\left(\mathrm{B}_{1}\right)$ by Laplace 's method in the similar way and continuing this method $(m-2)$ times, we have

$$
\begin{aligned}
\operatorname{det}\left(\mathrm{B}_{1}\right) & =\prod_{i=2}^{m} \operatorname{det}\left(\left(x-\lambda_{i}(\mathrm{H})\right) \mathrm{I}_{n}-\lambda_{i}(\mathrm{H}) \mathrm{A}(\mathrm{G})\right) \\
& =\prod_{\substack{2 \leqslant i \leqslant m \\
1 \leqslant j \leqslant n}}\left(x-\lambda_{i}(\mathrm{H})\left(1+\lambda_{j}(\mathrm{G})\right)\right) .
\end{aligned}
$$

$A(G)$ is orthogonally diagonalizable, so we can easily see that

$$
\mathrm{M}_{1}=\operatorname{det}\left[\begin{array}{cc}
(x-r) \mathrm{I}_{n}-r \mathrm{D}(\mathrm{G}) & -\sqrt{m} \mathrm{I}_{n} \\
-\sqrt{m} \mathrm{I}_{n} & x \mathrm{I}_{n}-\mathrm{D}(\mathrm{G})
\end{array}\right]
$$


By Proposition [2.7,

$$
\begin{aligned}
\mathrm{M}_{1} & =\operatorname{det}\left(x \mathrm{I}_{n}-\mathrm{D}(\mathrm{G})\right) \cdot \operatorname{det}\left((x-r) \mathrm{I}_{n}-r \mathrm{D}(\mathrm{G})-m\left(x \mathrm{I}_{n}-\mathrm{D}(\mathrm{G})\right)^{-1}\right) \\
& =\operatorname{det}\left(x \mathrm{I}_{n}-\mathrm{D}(\mathrm{G})\right) \cdot \prod_{i=1}^{n}\left(x-r-r \lambda_{i}(\mathrm{G})-\frac{m}{x-\lambda_{i}(\mathrm{G})}\right) \\
& =\prod_{i=1}^{n}\left(x-\lambda_{i}(\mathrm{G})\right) \cdot \prod_{i=1}^{n} \frac{x^{2}-\left((r+1) \lambda_{i}(\mathrm{G})+r\right) x+r \lambda_{i}^{2}(\mathrm{G})+r \lambda_{i}(\mathrm{G})-m}{x-\lambda_{i}(\mathrm{G})} \\
& =\prod_{i=1}^{n}\left(x^{2}-\left((r+1) \lambda_{i}(\mathrm{G})+r\right) x+\left(r \lambda_{i}^{2}(\mathrm{G})+r \lambda_{i}(\mathrm{G})-m\right)\right) .
\end{aligned}
$$

The proof of the next theorem is omitted because of similarity.

Theorem 4.2. The adjacency spectrum of $N_{\text {ex }}(\mathrm{G} \star \mathrm{H})$ is:

i) $\lambda_{i}(\mathrm{H})\left(1+\lambda_{j}(\mathrm{G})\right)$ for $i=2, \ldots, m$ and $j=1, \ldots, n$;

ii) $\left((r+1) \lambda_{j}(\mathrm{G})+r \pm \sqrt{\left.\left((r-1) \lambda_{j}(\mathrm{G})+r\right)^{2}+4 m \lambda_{j}^{2}(\mathrm{G})\right)} / 2\right.$ for $j=1, \ldots, n$.

\section{NeW Classes of integral GRAPHS}

In this section, we construct infinitely many families of integral graphs. The next theorem follows from Theorem B.], 3.2, 4.1] and 4.2].

Theorem 5.1. suppose that $\mathrm{G}$ is an integral graph with $n$ vertices and $\mathrm{H}$ is a r-regular integral graph with $m$ vertices, then

(1) The graph $I_{e x}(\mathrm{G} \circ \mathrm{H})$ is integral if $m=h(h \pm r)$ for some positive integer $h$.

(2) The graph $I_{e x}(\mathrm{G} \star \mathrm{H})$ is integral if for every $j=1,2, \cdots, n, m \lambda_{j}^{2}(\mathrm{G})=h(h \pm r)$ for some positive integer $h$.

(3) $N_{\text {ex }}\left(\mathrm{G} \circ p \mathrm{~K}_{2}\right)$ is integral graph if $8 p+1$ is perfect square.

(4) $N_{\text {ex }}\left(\mathrm{G} \star p \mathrm{~K}_{1}\right)$ is integral graph if $4 p+1$ is perfect square.

As an special case of previous theorem, we have the following corollary.

Corollary 5.2. suppose that $\mathrm{G}$ is an integral graph, then for each positive integer $p$ and $q$,

(1) $I_{e x}\left(\mathrm{G} \circ \mathrm{K}_{p}\right), I_{e x}\left(\mathrm{G} \circ p \mathrm{~K}_{p, p}\right)$ and $I_{e x}\left(\mathrm{G} \circ \mathrm{C}_{3}\right)$ are integral graphs.

(2) $I_{e x}\left(q \mathrm{~K}_{2} \star \mathrm{K}_{p}\right)$ and $I_{e x}\left(q \mathrm{~K}_{2} \star p \mathrm{~K}_{p, p}\right)$ are integral graphs. 
Alg. Struc. Appl. Vol. 5 No. 2 (2018) 23-34.

Remark 5.3. In Theorem 5.0 and Corollary 5.2, one can replace G with any graph of the classes of integral graphs has obtained so far. in fact, all the integral graphs obtained from new operations on the graphs can replace by G; for example, one can see the class of integral graphs obtained in [1, 2], 16, 17].

\section{Question}

Regarding the concept of permutation and definition of identity-extended corona and identity-extended neighborhood corona, it seems that this concept can be generalized to the $\sigma$-extended corona and $\sigma$-extended neighborhood corona. It seems that such an extension of the corona corresponds to many molecular bonds, and thus by obtaining a variety of spectra of such expansion, some of the indices associated with those bonds would be calculated. But this question still remains open, how can obtain these spectrums?

\section{ACKNOWLEDGMENTS}

The authors wish to sincerely thank the referees for several useful comments.

\section{REFERENCES}

[1] Ch. Adiga, B.R. Rakshith and K.N. Subba Krishna, Spectra of extended neighborhood corona and extended corona of two graphs, Electronic Journal of Graph Theory and Application. Vol. 4 No. 1 (2016), pp. 101-110.

[2] Ch. Adiga, B.R. Rakshith and K.N. Subba Krishna, Spectra of some new graph operations and some new classes of integral graphs, Iranian Journal of Mathematical Sciences and Informatics. Vol. 13 No. 1 (2018), pp. 51-65.

[3] K. Balińska, D. Cvetković, Z. Radosavljević, S. Simić, D. Stevanović, A survey on integral graphs, Univerzitet u Beogradu. Publikacije Elektrotehnickog Fakulteta. Serija Matematika. Vol. 13 (2002), pp. 42-65.

[4] S. Barik, S. Pati and BK. Sarma, The spectrum of the corona of two graphs, SIAM Journal on Discrete Mathematics. Vol. 21 (2007), pp. 47-56.

[5] S. Barik, G. Sahoo, On the Laplacian spectra of some variants of corona, Linear Algebra and its Applications. Vol. 512 (2017), pp. 32-47.

[6] L. Barriere, F. Comellas, C. Dalfo and M. A. Fiol, The hierarchical product of graphs, Discrete Applied Mathematics. Vol. 157 (2009), pp. 36-48.

[7] AE. Brouwer, WH. Haemers, Spectral of graphs, Springer, New York, (2012).

[8] S.-Y. Cui, G.-X. Tian, The spectrum and the signless Laplacian spectrum of coronae, Linear Algebra and its Applications. Vol. 437 (2012), pp. 1692-1703.

[9] D. B. S. Cvetković, M. Doob and H. Sachs, Spectra of graphs- theory and applications, (Third edition), Johann Ambrosius Barth, Heidelberg, (1995).

[10] D. B. S. Cvetković, P. Rowlinson and H. Simić, An introduction to the theory of graph spectra, Cambridge University Press, Cambridge, (2010).

[11] W. L. Ferrar, A text-book of determinants, matrices and algebraic forms, (Second edition), Oxford University Press, (1957). 
[12] I. Gutman, The energy of a graph, Berichte der MathematischStatistischen Sektion im Forschungszentrum Graz. Vol. 103 (1978), pp. 1-22.

[13] F. Harary, Graph theory, Narosa Publishing House, (1969).

[14] R.A. Horn, C.R. Johnson, Topics in matrix analysis, Cambridge University Press, New York, (1991).

[15] Y. -P. Hou, W. -C. Shiu, The spectrum of the edge corona of two graphs, Electronic Journal of Linear Algebra. Vol. 20 (2010), pp. 586-594.

[16] G. Indulal, New families of integral graphs, Discrete Mathematics, Algorithms and Applications. Vol. 7 No. 4 (2016), 1650063 (9 pages).

[17] G. Indulal, Spectrum of two new joins of graphs and infinite families of integral graphs, Kragujevac Journal of Mathematics. Vol. 36 (2012), pp. 133-139.

[18] J. Lan, B. Zhou, Spectra of graph operations based on R-graph, Linear and Multilinear Algebra. Vol. 63 No. 7 (2015), pp. 1401-1422.

[19] Xi. Liu, P. Lu, Spectra of subdivision-vertex and subdivision-edge neighbourhood coronae, Linear Algebra and its Applications. Vol. 438 (2013), pp. 3547-3559.

[20] Xi. Liu, Z. Zhang, Spectra of subdivision-vertex join and subdivision-edge join of two graphs, Bulletin of the Malaysian Mathematical Sciences Society. (2017) https://doi.org/10.1007/s40840-017-0466-z

[21] P.-L. Lu, Y.-F. Miao, Spectra of the subdivision-vertex and subdivision-edge coronae, arXiv:1302.0457.

[22] C. McLeman, E. McNicholas, Spectra of coronae, Linear Algebra and its Applications. Vol. 435 (2011), pp. 998-1007.

[23] Y. Qi, H. Li, Zh. Zhang, Extended corona product as an exactly tractable model for weighted heterogeneous networks, The Computer Journal. Vol. 61 No. 5 (2018), pp. 745-760.

[24] S.-L. Wang, B. Zhou, The signless Laplacian spectra of the corona and edge corona of two graphs, Linear and Multilinear Algebra. Vol. 61 No. 2 (2013), pp. 197-204.

[25] F.-Z. Zhang, The Schur complement and its applications, Springer, (2005).

\section{Maliheh Tajarrod}

Department of Mathematics

Kerman Branch, Islamic Azad University

Kerman, Iran.

malihetajarrod2@gmail.com

\section{Tahereh Sistani}

Department of Mathematics

Kerman Branch, Islamic Azad University

Kerman, Iran.

taherehsistani@gmail.com 